\title{
Learning, investments and derivatives
}

\author{
Andrei N. Soklakov
}

August 12, 2010*

\begin{abstract}
The recent crisis and the following flight to simplicity put most derivative businesses around the world under considerable pressure. We argue that the traditional modeling techniques must be extended to include product design. We propose a quantitative framework for creating products which meet the challenge of being optimal from the investors point of view while remaining relatively simple and transparent.
\end{abstract}

\section{Introduction}

In the last few decades, the world of financial derivatives experienced a true explosion in the development of quantitative methods. Remarkably, very little of that development went into supporting the process of product innovation and structuring - the cornerstone of every derivative business! Compared to modeling, which continues to attract most quantitative effort, product innovation remains essentially a form of art. Without diminishing the importance of accurate modeling, it is equally important to recognize that payoff structure of a financial product is not just a source of modeling challenges, - it is a central part of a quantitative solution, - a part which needs its own quantitative support. Innovation going badly has been among the main themes of the recent crisis. The time has come for a serious look at quantitative ways of making the process of innovation more rational and systematic. The purpose of this article is to introduce some elementary arguments which may form the basis of a more rational quantitative approach to structuring.

For the sake of clarity and generality we develop our key arguments from first principles. Going back to the beginning, we see two basic reasons for trading derivatives. First one is the demand for bespoke hedging solutions. The other is leveraged investments. Without jumping to conclusions as to which of these two reasons is more or less natural, it would be fair to say that the recent crisis raised serious concerns about the use of derivatives for investment purposes. For this reason we investigate structuring investments as our first priority.

Investment decisions are driven by peoples' beliefs as much as by economic facts. We therefore start by looking for a simple mathematical language that treats facts and beliefs on an equal footing. We show how beliefs can be converted into tradable payoff structures

\footnotetext{
*Date of the original submission to RISK Magazine.
} 
and, conversely, how to imply investors beliefs from the payoff structures they use. This gives us tools for tailoring new products as well as checking implicit assumptions behind existing positions.

Most people would agree that complex products can be dangerous. What is less obvious is that simple products can also be pretty far from ideal. We find that investment products can be very easily oversimplified to the point when they can no longer reflect any rational view. Paraphrasing the famous observation about physical theories, investments should be made as simple as possible but not simpler.

Two fundamental features distinguish investment from speculation: the use of in-depth research and attention to safety. The above caution on oversimplification is related to the ability of products to accommodate results of in-depth research. Thinking about safety, we show how one can use the technology of derivatives to stabilize raw investment strategies. This line of thinking leads us to a class of derivatives which, in terms of their complexity, lie between vanillas and exotics.

\section{Investments and probability distributions}

Consider a random variable representing an observable market parameter: stock price, index value, interest rate, etc. Everything we know about the variable can be summarized using probability distributions. The ability of probability distributions to summarize the knowledge about random variables does not depend on whether this knowledge is factually true or not. This powerful feature of probability-based descriptions left its mark on most modern fields of human knowledge: from foundations of quantum mechanics to artificial intelligence. It is certainly very important for describing the world of finance where both hard data and investors' beliefs have material impact.

Interplay between reality and beliefs is at the heart of every investment. We set the stage by introducing distinct concepts of market-implied, investor-believed and actual realized distributions - all of which refer to the same market variable. These distributions play three distinct roles which can be outlined as follows. Market-implied distribution, $m()$, represents the market view. The reader is almost certainly familiar with deriving such distributions from the relevant market prices. The investor's view is given by the believed distribution, $b()$. The form of this distribution is entirely up to the investor and we do not make any assumptions regarding $b()$. Realized distribution, $p()$, represents the reality that both the market and the investor are trying to predict. In practice, realized distribution can be very difficult to estimate leading to the use of proxies such as realized mean and realized variance. We use $m(), b()$ and $p()$ as auxiliary concepts - as thinking tools - refining the definitions along the way as required. We shall see, however, that these concepts have very real and easily accessible (often visualizable) meaning.

In hindsight, it is not surprising to find products which already use the language of distributions in their intuition. Futures and variance swaps, for example, compare the mean and the variance of the market-implied and realized distributions. Exotics, such as barriers, suggest that there might be more to trading distributions than using a few of their leading moments. This is indeed the case. Imagine, for example, that you have strong opinions on both the mean and the variance of some market variable. How would 
you allocate your money? Should you put most of it on the mean or on the variance? Are you even sure that your opinion is better expressed with two separate trades (one on the mean and the other on the variance), or is there a structured product that is optimized to express the combined view? We shall see that thinking in terms of probability distributions makes such questions trivial and to a large degree redundant.

\section{Growth-optimizing investor}

One can talk about optimality of investments only with respect to an objective. As illustrated by Markowitz, different objectives normally lead to different optimal investments. On the practical side this means a high focus on examples. The aim of this section is to introduce an example of a particular investor which is then used as a reference point supporting the arguments presented in the subsequent sections. The case of a general investor is presented in [1].

Imagine an investor who considers himself an expert on a certain market variable. The investor is very confident in his expertise and is seeking a buy-and-hold product with two characteristics: the product must make use of the entire expert knowledge about the variable and offer the highest expected rate of return. Even without formal analysis it is clear that this growth-optimizing investor is very aggressive. With a possible exception of near-crisis bubble periods, when the rate of returns becomes a common obsession, most people would be more risk averse.

Let us start our analysis with the simplest limiting case. Imagine that the investor is absolutely sure that the value of the market variable, $x$, at certain future time will lie in a small interval $\left[K_{1}, K_{2}\right]$. Graphically, this belief can be described by the probability distribution on Fig. 1A. The optimal investment strategy expressing this belief must offer nonzero reward if $x$ falls within the interval and pay zero otherwise. For small enough $\delta=K_{2}-K_{1}$ the variation of the payment within the interval can be neglected and the whole strategy can be viewed as buying a digital call spread with strikes $K_{1}$ and $K_{2}$.

In a slightly more general case, the investor might believe that two small intervals $\left[K_{1}, K_{2}\right]$ and $\left[L_{1}, L_{2}\right]$ contain the future outcome for $x$. This belief can be summarized by a pair of probabilities $p_{K}$ and $p_{L}=1-p_{K}$ as illustrated by the distribution on Fig. 1B. As before, it is clear that the investor should focus on combinations of two binary spreads. The only nontrivial question here is how to split the investment between the two spreads in order to maximize investor's utility.

In general, one can imagine a fine set of binary spreads covering the domain of any reasonable probability distribution (Fig. 1C.). The problem of optimal investment strategy (maximum expected utility) can then be formulated as the problem of finding proportions of the total investment which should be allocated to each of the binary spreads.

At the mathematical level, the above discussion connects the optimal investment problem to the classic analysis of horse racing scenarios: just think of the binary spreads as "horses", with only one of them "winning" i.e. maturing in the money. The mathematics of such scenarios has been around for a long time and is very well understood. In what follows we use this understanding without assuming any special expertise from 
the reader 1

1 Believed distribution and capital allocation via binary spreads

(A)

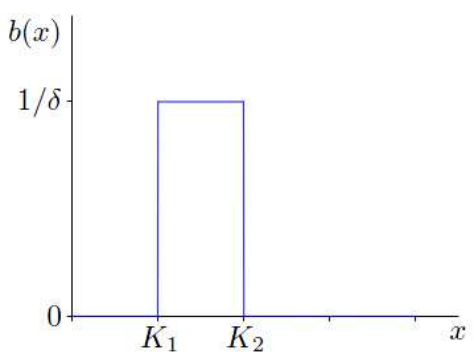

(B)

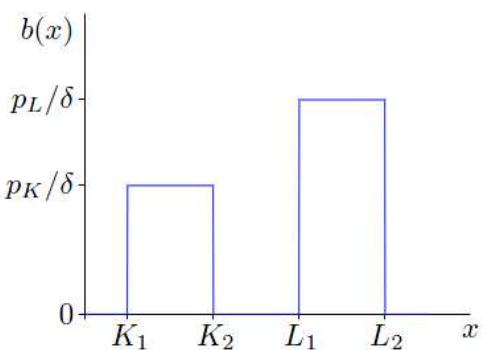

(C)

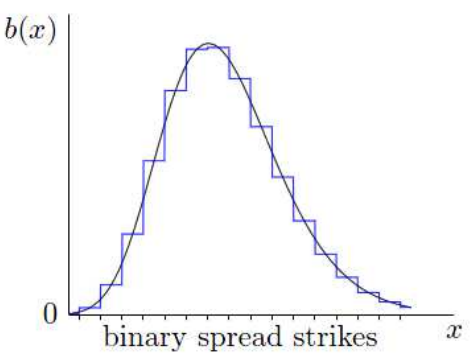

Note: the domain of any believed distribution can be partitioned into small intervals and any investment can then be viewed as allocation of capital across the corresponding binary spreads (see the main text).

Let $N$ be the number of binary spreads and let $\left\{R_{i}\right\}_{i=1}^{N}$ be the returns on each of the spreads offered by the market. Furthermore, let $\left\{\alpha_{i}\right\}_{i=1}^{N}$ be the proportions in which the investor chooses to split their investment across the spreads $\left(\sum_{i} \alpha_{i}=1\right)$. If $b_{i}$ is the investor's believed probability that the $i$ th spread matures in the money, then for the expected rate of return he would compute

$$
\mathbb{E}_{b}[\text { rate }]=\sum_{i} b_{i} \ln \left(\alpha_{i} R_{i}\right)
$$

Maximizing this over all possible $\left\{\alpha_{i}\right\}_{i=1}^{N}$ subject to the constraint $\sum_{i} \alpha_{i}=1$ leads us to the classic result [2]

$$
\alpha_{i}^{*} \stackrel{\text { def }}{=} \text { optimal } \alpha_{i}=b_{i}
$$

In other words, investment in proportion to the believed probabilities maximizes the expected rate of return (all from the investor's point of view). In what follows we will also need the expression for the actual expected return from this strategy. This is

$$
\mathbb{E}_{p}[\text { rate }]=\sum_{i} p_{i} \ln \left(\alpha_{i}^{*} R_{i}\right)=\sum_{i} p_{i} \ln \left(b_{i} R_{i}\right)
$$

where $p_{i}$ are the actual realized probabilities which are unknown at the time of investment. For simplicity, we start by looking at the "fair odds" case when $\sum_{i} 1 / R_{i}=1$. This allows

1 Given the sensitivity of the post-crisis time of this publication, it is worth emphasizing that our references to the mathematics of horse racing are purely pedagogical (as is, for example, the famous Prisoner's Dilemma in decision theory). There is no doubt that the relevant pieces of logic can be introduced without any reference to gambling (or prisoners). Such references however export the clarity of mathematical understanding to a very wide audience - a feature worth keeping if we are to achieve maximum transparency. 
us to define the market-implied probability, $m_{i} \stackrel{\text { def }}{=} 1 / R_{i}$, that the $i$ th spread matures in the money and rewrite the above formula as

$$
\mathbb{E}_{p}[\text { rate }]=\sum_{i} p_{i} \ln \frac{b_{i}}{m_{i}} .
$$

In the above derivation of equations (14-4) we chose to make two simplifying assumptions: all of the investment is allocated across the spreads (as opposed to keeping some of it as cash) and $\sum_{i} 1 / R_{i}=1$. We did this as a logical shortcut which makes mathematics throughout this paper particularly transparent. In the Appendix we show what happens when these assumptions are removed: some interesting subtleties emerge but the core logic remains intact. The reader is advised to continue with the main text of the paper before reading the Appendix.

Speaking about assumptions, it is important to note that we do not use any pricing or econometric models. For us, market-implied probabilities are nothing but a probabilistic way of recording market returns. It is just like probabilistic interpretation of betting returns - the odds. And just like people on the race track, who discuss the odds offered by the bookie, we can speak about $m_{i}$ as long as the market offers the relevant prices. This remains true even if the market is distorted by inefficiencies, including arbitrage and large bid-offer spreads. Our definition of market-implied distribution is therefore valid even when a closely related concept of market-implied pricing measure does not exist. Investor's belief probabilities, $b_{i}$, will be discussed in detail in the next section. We will not need the realized distribution, $p_{i}$, until the penultimate section of the paper.

\section{The maximal payoff of learning}

In terms of probabilities, the process of learning is often described via Bayes' theorem

$$
P(x \mid \text { research })=\frac{P(\text { research } \mid x)}{P(\text { research })} P(x),
$$

which tells us how the prior knowledge $P(x)$ about random variable $x$ is updated to $P(x \mid$ research $)$ on the account of learning from results of additional research. This formula follows immediately from the properties of conditional probabilities and is therefore valid for any consistent learning process regardless of whether or not the actual implementation makes use of Eq. (5).

In the previous section we computed the return to the growth-optimizing investor as

$$
\alpha_{i}^{*} R_{i}=b_{i} R_{i}=\frac{b_{i}}{m_{i}}
$$

where $i$ is the index of the binary spread which ends up maturing in the money. Considering this index as a function of the underlying market variable, $i=i(x)$, we can write the payoff (6) from a single run of the growth-optimizing strategy as

$$
f(x)=\frac{b(x)}{m(x)},
$$


where $b(x)=b_{i(x)} / \Delta x$ and $m(x)=m_{i(x)} / \Delta x$ are the believed and the market-implied probability densities. Rearranging the terms, we obtain

$$
b(x)=f(x) m(x) .
$$

Comparison of this formula to Eq. (5) gives us the fundamental connection between the payoff structure for the optimal growth strategy and the underlying learning process. Indeed, any rational investor starts by looking at market prices. Mathematically, this means that the investor is taking the market-implied view as the prior, $P(x)=m(x)$. Any logical alternative to this choice of prior would imply mistaking market information. The investor then updates the prior by performing, purchasing or assuming additional research. This step may or may not use Bayes' theorem in an explicit way. However, if the investor is capable of writing the updated view as a probability distribution, it would

have to be the posterior belief $b(x) \stackrel{\text { def }}{=} P(x \mid$ research $)$. This leaves us no choice but to recognize Eq. (8) as a special case of Eq. (5). The general case of an arbitrary investor is subject to the same logic and is equally straightforward to obtain [1].

The above connection between the process of learning and the associated optimal investment growth is interesting in its own right. For the sake of brevity we focus on just a couple of practical observations which are important for the conclusions of this paper and leave the rest for future discussions. The observations we have in mind can be formulated as follows.

o1 Investor's view, $b(x)$, and the the shape of the growth-optimal payoff, $f(x)$, can be computed from each other (using Eqs. (8) and (7) respectively).

o2 Growth-optimal payoff $f(x)$ is a likelihood function: it is proportional to the conditional probability $P($ research $\mid x)$.

Observation o1 allows us to provide the growth-optimizing investor with a payoff structure that matches their view. More importantly, this observation allows us to analyze any payoff function $f(x)$ by looking at the view $b(x)$ that is associated with it. This can be useful even if the actual investor is not growth-optimizing. In the next section we give some examples illustrating this point and show how observation o2 can be used for additional sanity checks.

\section{Payoff analysis}

Before looking at example payoffs, let us outline some basic features that we expect to see in a rational buy-and-hold investment. Imagine a growth-optimizing investor who did no research that would challenge the prevailing market view. Such an investor cannot justify any view that would be materially different from $m(x)$. We must therefore have $b(x)=m(x)$ and $f(x)=1$ for any $x$. In other words, without adequate research any investment which is different from the risk-free $f(x) \equiv 1$ would be nothing but a pure gamble - a gamble which cannot be justified even by the relatively aggressive growthoptimizing investor. 
Imagine now that the investor did some research acquiring additional knowledge about the market variable in the interval $a \leq x \leq b$. The believed distribution, $b(x)$, will now be different from the market-implied, $m(x)$, although the two should still agree outside the interval

$$
\forall x \notin[a, b]: b(x)=m(x) \Rightarrow f(x)=1 .
$$

Figure 2 illustrates a possible payoff structure satisfying this condition.

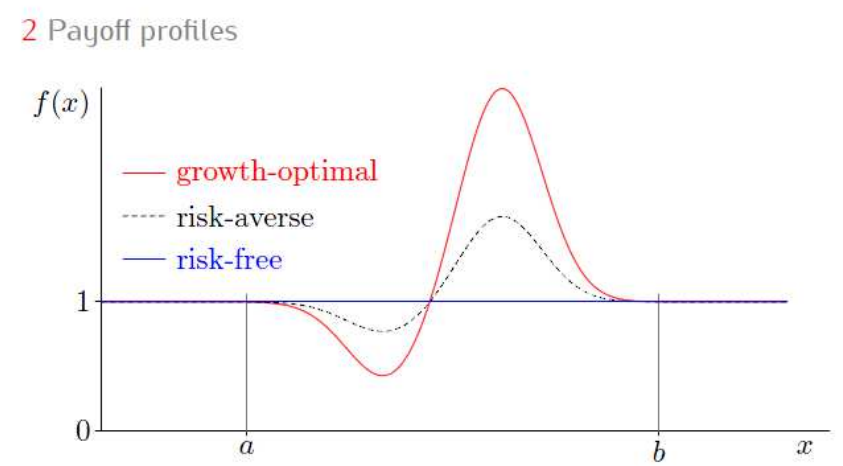

Note: payoff example expressing a bullish view on $x$. Qualitative comparison of payoff functions with the same underlying research but with different degree of risk aversion: all payoffs coincide with the risk-free outside the researched domain; most risk-averse investors would choose a payoff between the growth-optimal and the risk free profiles.

How would this payoff structure change if the investor was not growth-optimizing? As mentioned before, most investors would be more risk averse. Achieving greater risk aversion across all values of $x$ is possible only if we chose a payoff that is closer to the risk free line, $f(x) \equiv 1$, at every $x$. It follows that the payoff favored by most investors (from zero risk appetite to that of the growth-optimizing investor) must lie between the growth-optimal and the risk-free payoffs as illustrated on Fig. 2.

It is worth mentioning that there are limits to every research and the mathematical extremes of market variables are all but guaranteed to fall outside these limits. This means that some analogue of boundaries $a$ and $b$ introduced above would always apply in practice. What can happen if we ignore this fact and extrapolate our payoff beyond the boundaries of research? To answer this question consider a market which moved outside the boundaries and an investor who just realized that his positions correspond to a view that he never researched and therefore never intended to express. Cashflows become a matter of luck. Not many scenarios can compare to that in terms of potential for panic, disappointment and embarrassment on all sides of the relevant trades.

\section{Simple payoffs}

Looking at Fig. 2 we cannot help noticing that very few simple products can be tuned to comply with the boundary requirement (9). This is not a good sign. Indeed, we recall that most simple products were never really designed as investment vehicles. The original 
vanillas, for example, were clearly about hedging - ensuring businesses on future prices of goods and supplies. Investors do however explore all available markets. Moreover, in times of uncertainty investors naturally flee towards established hedging markets. It is therefore prudent to include classic simple payoffs into our analysis - especially in the current climate when the regulatory pressure amplifies and prolongs the natural flight to simplicity.

Figure 3 shows the relationship between market-implied and investor-believed distributions which correspond to the at-the-money European call and digital options. We see that investor's beliefs must be pretty far from the market consensus to justify such products as buy-and-hold investments. We leave the reader to judge how extreme investor-implied beliefs can become. We note only that in the case of out-of-the-money options meaningful display of market-implied and investor-believed distributions on the same plot becomes, typically, impossible without introducing separate plotting scales.

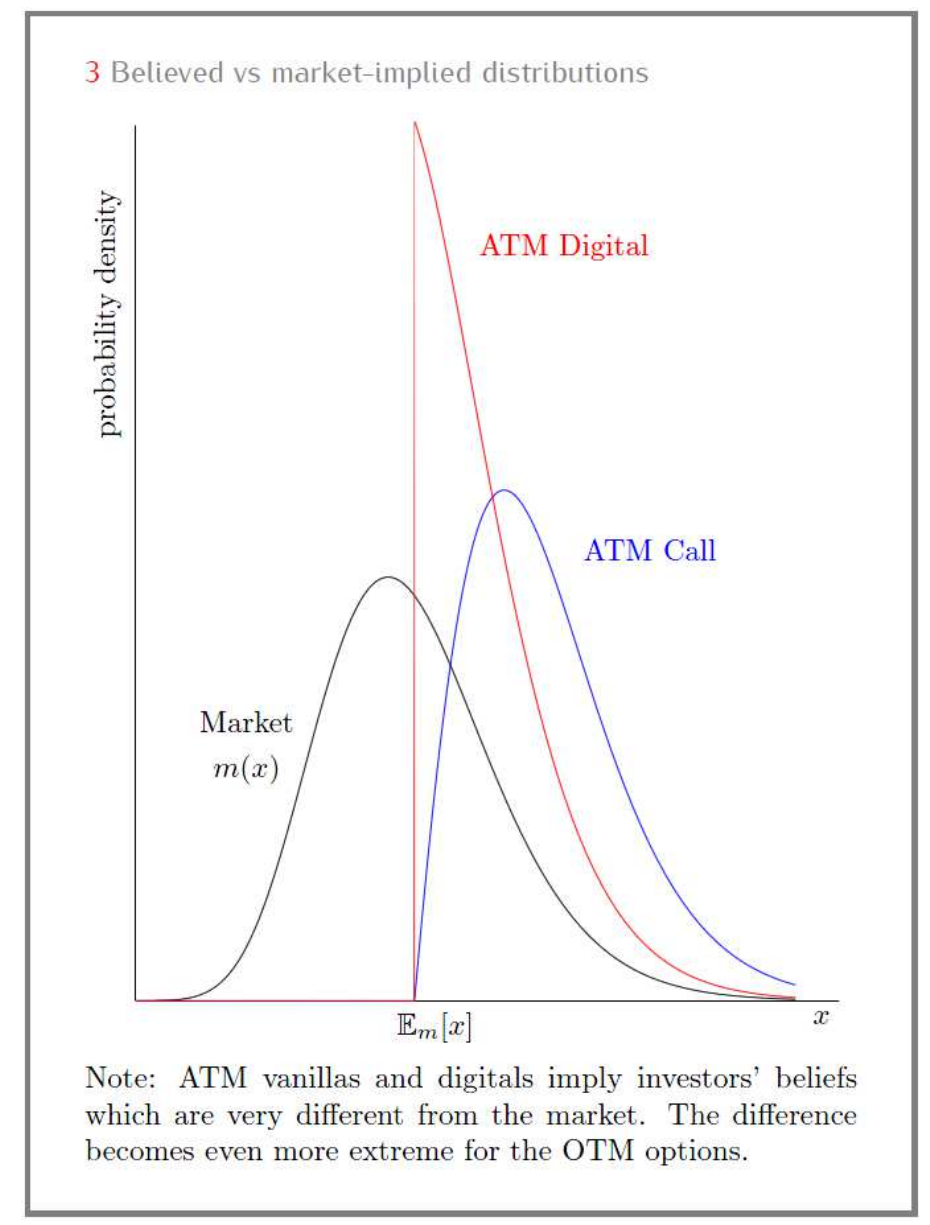

Visual comparison of distributions is informative when the probabilities in question are reasonably large. When it comes to judging rare events, however, human intuition is notoriously poor. Looking at the tails on Fig. 3, for example, we see that buying an at-the-money call option implies a view which is likely to be over-leveraged on the right tail. It does not look as dramatic though as the deviation from the market view near at-the-money. Experience tells us that visual inspection of the tails may not give us the whole story. Formal reasoning is required. 
A more formal approach to analyzing payoff structures can be developed from their interpretation as likelihood functions (see observation o2 above). Note, for example, that realistic investors cannot possibly claim that their research provides an unbounded amount of useful information. Most of them are still confined to the decision paradigm described by just a few possible outcomes (e.g. "buy", "hold" or "sell"). In mathematical terms this means that "research" in Eq. (5) belongs to a discrete finite set of outcomes. This implies that $P($ research $\mid x)$ is bounded and $f(x)$ cannot diverge to infinity. We conclude that anyone with an initially neutral position who uses a call option as a buy-and-hold investment is behaving in an economically indistinguishable way from a growth-optimizing investor with views so extreme that no research could be found to back them up (without contradicting the basic laws of probabilities).

Despite all we have said above, vanilla products are perceived by investors as a relatively safe choice among derivatives. This is because of the huge hedging market which is formed by these products. Liquidity provided by the market allows investors to dump their investments without having to wait until maturity. Liquidity is a good thing to have, but excessive substitution of liquidity in place of prudent investment design is dangerous. We have seen that the use of vanillas as investment vehicles implies rather extreme views. Widespread expression of such views signals expectation of instability. This expectation must get reflected by the market: after all, we are talking about real investors placing real money on extreme market events. In the limiting case we could end up with a crash, but in any case the market should price investors' behavior and we should see fattening of the tails in the return distributions. 2

\section{Information derivatives}

In this section we touch a bit more upon the question of safety. An honest investor should be prepared to make some losses (in real terms) if their view turns out to be less accurate than the market. A lot of statistical trading strategies, however, can lead to losses even if the underlying view is exactly right. You may, for example, hold the perfectly accurate belief that an unbiased coin flip has $1 / 2$ chance of producing heads or tails and still struggle to stay in the game through a particularly unlucky sequence of coin flips. In this section we present an idea for how this kind of risk can be reduced with the help of derivative technologies.

It can be shown that the expected performance of the growth-optimizing strategy can be achieved in the long run by simple repetition: by reinvesting proceeds from one investment into the next 22. This, of course, is rather risky in practice. Instead of performing such reinvestments for real, we propose to compute the expected performance mathematically and use the resulting formula as a definition of a derivative product. Effectively, we propose to skip the bulk of risky and expensive reinvestments and, instead of waiting for the law of large numbers to kick in, we offer direct exposure to the expected payoff. Statistical (i.e. risky) convergence on the investor's side is thereby replaced with derivative

\footnotetext{
${ }^{2}$ Could this be what happened in the crash of 1987? Black-Scholes inspired technologies lead to unprecedented growth in speculative trading of vanilla products. Market crashed, but quickly stabilized around a new type of equilibrium with considerably fatter tails (volatility skew).
} 
hedging on the sell side. This provides added value to the investor and uses much safer technology (hedging vs.statistical convergence).

The easiest way to explain this idea is by giving an example. In the following we deliberately bring up a few important points from the previous sections to show how they all fit together. Consider a market which believes in a lognormal distribution for variable $x$

$$
m(x)=\operatorname{LN}\left(\mu, \sigma_{m}\right) \stackrel{\text { def }}{=} \frac{1}{x \sqrt{2 \pi \sigma_{m}^{2}}} \exp \left[-\frac{(\ln x-\mu)^{2}}{2 \sigma_{m}^{2}}\right] .
$$

Imagine now an investor who agrees with the market on everything apart from the variance which he believes to be underestimated by the market. This kind of view relative to the market is very plausible (if not common). Mathematically, the investor-believed distribution must be $b(x)=\operatorname{LN}\left(\mu, \sigma_{b}\right)$ where $\sigma_{b}>\sigma_{m}$. An attentive reader would immediately spot a problem: the growth-optimal payoff

$$
f(x)=\frac{b(x)}{m(x)}=\frac{\sigma_{m}}{\sigma_{b}} \exp \left[\left(\frac{1}{\sigma_{m}^{2}}-\frac{1}{\sigma_{b}^{2}}\right) \frac{(\ln x-\mu)^{2}}{2}\right]
$$

is unbounded in the wings contradicting Eq. (9). By observation o2, even the growthoptimizing investor would have to "bend the rules" of conditional probabilities to justify this kind of leverage in the wings. Let us proceed with this view anyway and see where it would lead us. This simple example gives us the opportunity to trace the propagation of over-leveraging in the wings from a seemingly innocent belief to the final product. We then discuss how this over-leveraging can be avoided or mended.

Substituting (11) into (44) and introducing the concept of realized variance $\sigma_{p}^{2}$ by writing $p(x)=\mathrm{LN}\left(\mu, \sigma_{p}\right)$ we obtain

$$
\mathbb{E}_{p}[\text { rate }]=\int_{0}^{\infty} p(x) \ln \frac{b(x)}{m(x)} d x \propto \sigma_{p}^{2}+\text { const },
$$

which is the payoff structure of a variance swap. With $\sigma_{b}>\sigma_{m}$, the coefficient before $\sigma_{p}^{2}$ is positive, suggesting the investor takes a long position in the swap.

Theoretically, variance swaps are among the best understood products. In practice, however, traders are all too familiar with difficulties that come with managing this product [3]. Although the above investment scenario is simplistic, our approach succeeds in raising a warning which turns out to be true in a much wider context. Moreover, it gives us the following high-level explanation of the problem. Variance is a global characteristic of a distribution. Consequently, variance swaps express a view across the whole of the distribution including the wings. This includes extreme values of $x$ which fall outside any finite range covered by research. On the hedging side this looks like exposure to all strikes from zero to infinity - including the strikes which fall outside the finite range available for practical hedging. No amount of traditional modeling is going to change this fact and the solution to such problems is clearly in the domain of product design.

Indeed, there is now a variety of modifications to the basic variance swap which attempt to address the shortcomings of the original design (see, e.g. 44). Capped, conditional, corridor and gamma swaps have been introduced. Index variance swaps have been the longest used example of the original payoff structure. They too have recently developed 
caps on the account of hedging problems that became apparent during the last crisis. For single-stock variance swaps such caps proved to be insufficient and the market is once again exploring alternative payoff structures [5]. The market is clearly looking for prudently designed investment structures but, to our shame, realized losses are still setting the trends of this development.

As the above discussion shows, we must get into the habit of designing products which acknowledge the limits of available information. This can be done either explicitly, by checking the asymptotic behavior (9), or via payoff-related modeling, as considered in [6]. These two approaches are of course related: they both rely on concepts of information and information processing and even lead to some similar product designs. The reason for this is very simple: for investment purposes, information is the ultimate asset class. Information production, processing and packaging into financial products are just natural facets that we must examine when building, using and regulating the use of investment products.

\section{Summary and outlook}

We presented a framework for creating new and analyzing existing buy-and-hold investment structures. We did that using very simple arguments and hardly any mathematics. As a result, all product complexity becomes directly linked to that of investor's research, market data and, if you want to use the technique of the last section, the general form of a future realized distribution. No exotic features would appear in the final product unless they are evident from investor's research or market data. Barriers and digitals, for example, would not appear unless investor's beliefs or market data were best described by discontinuous probability distributions. In spite of this push to simplicity, we find that very simple payoffs can struggle to match realistic beliefs of a buy-and-hold investor. Judging by [6], the complexity of basic information derivatives is similar to that of third generation volatility products (e.g. gamma swaps).

On the technical side we show that optimal payoff structures are intimately connected to the central concept in statistical inference, - the likelihood functions. This can be useful for transforming results of statistical research into tradable payoff structures. In terms of tools for further research, information theory, learning theory and utility framework should provide the core technical concepts.

In this paper we steered as far away from modeling as we could. This was done to emphasize the importance of model-independent aspects of product design. Models, however, do influence the design of investment products. Our framework is well positioned for analyzing such influence. More generally, our approach can be applied to rationalizing the use of different models. Indeed, a calibrated model is nothing but a sophisticated definition of a probability distribution. Models can be used to express a view on the market just like the probability distributions that we considered above. We can expose model differences by designing products that exploit them. This is of course likely to require more advanced analysis, but only as advanced as the models in question. Finally, our approach may add motivation and ideas to modeling approaches based on information processes (see e.g. [7]). 
Andrei Soklakov is a Vice President in Model Risk and Analytics of Deutsche Bank. He would like to thank his former and present colleagues at both Deutsche Bank and Goldman Sachs for their interest in this work. The core setup underlying the results of this paper was first presented by the author at Quant Congress Europe 2008. The views expressed herein should not be considered as investment advice or promotion. They represent personal research of the author and do not necessarily reflect the view of his employers, or their associates or affiliates. Email: Andrei.Soklakov@db.com, Andrei.Soklakov@gmail.com.

\section{Appendix}

Equation (8) and its interpretation in terms of Bayesian learning is probably the most important logical element of this paper. We arrived at it by considering a growthoptimizing investor and by making two further assumptions: all of the capital is invested and $\sum_{i} 1 / R_{i}=1$. It turns out that none of these assumptions are necessary. Somewhat amazingly, the same core logic holds even in the case of an arbitrary rational investor. The case of general investors requires a separate presentation and we will provide it in a follow-up paper [1]. Here we give a brief summary of what happens if we remove all additional assumptions in the case of growth-optimizing investor.

If all of the investment capital is committed, $\sum_{i} \alpha_{i}=1$, then the optimization problem, Eqs.(112), does not depend on whether $\left\{1 / R_{i}\right\}$ add up to one or not. The only thing that needs fixing is the normalization of market-implied probability. To this end we compute the reference return, $R$, by setting $1 / R=\sum_{i} 1 / R_{i}$ and replace the previous definition

of the market-implied probability with a more general one: $m_{i} \stackrel{\text { def }}{=} R / R_{i}$. All arguments follow as before and Eq. (8) becomes

$$
b(x)=\frac{f(x)}{R} m(x) .
$$

This leaves intact the interpretation of the payoff, $f(x)$, as the likelihood function (although, of course, one should be careful with numerical details: payoff asymptotics, etc.).

We now remove the remaining assumption and allow the investor to keep some of the investment capital risk-free (growing at the rate $R_{0}$ ). This becomes relevant when $R<R_{0}$, i.e. when the risky returns offered by the market appear less attractive than risk-free. Mathematically, this case is very similar to the case of "unfair odds" (see Ref. [2]) - we just need to introduce the concept of risk-free rate, $R_{0}$, which is not present in the classic betting paradigm. As expected, the investment becomes a sum of risky investments on some values of $x$ (lets denote them $\omega$ ) and a risk-free floor. We derive

$$
b(x)=\frac{f(x)}{R_{0}} m^{*}(x), \quad \text { and } m^{*}(x) \stackrel{\text { def }}{=} \begin{cases}R_{0} / R_{i(x)}, & x \in \omega, \\ b(x) / \alpha_{0}, & x \notin \omega,\end{cases}
$$

where $m^{*}(x)$ is a probability distribution and the normalization constant $\alpha_{0}$ coincides with the fraction of the capital invested in a risk-free bond. The case $x \in \omega$ is as clear as Eq. (13). To understand what happens for $x \notin \omega$, imagine coming to a fruit market and 
noticing an ordinary apple being on sale for one billion dollars. You cannot accept that

price even as prior information for your research. Similarly, investment market for $x \notin \omega$ does not really exist - the risky returns are far to low (as compared to risk-free). The market simply does not offer any reasonable prior view and the investor patches it with the only view available - his own belief. In terms of the payoff for $x \notin \omega, b(x)$ drops out of Eq. (14) leaving the equation for the risk-free payoff $f(x)=\alpha_{0} R_{0}$ (just like it should be in all cases when the investor's research cannot proceed). We see that our framework makes sense even when investment markets struggle to exist.

\section{References}

[1] Soklakov, A., forthcoming.

[2] Kelly J L Jr, "A New Interpretation of Information Rate", Bell System Technical Journal, 917-26 (1956).

[3] Chriss N and W Morokoff, "Market risk for volatility and variance swaps", Risk, October (1999), pp 55-59.

[4] Carr P and K Lewis, "Corridor variance swaps", RISK, February (2004), pp 67-72.

[5] Clark J, "Return to variance?", RISK, February (2010), pp. 65-67.

[6] Soklakov, A., "Information Derivatives", RISK, April (2008), pp. 90-94.

[7] Brody D and R Friedman, "Information of interest", RISK, December (2009), pp 101-106. 